\title{
Comparison of outcome after sentinel lymph node biopsy or inguinal lymph node dissection in patients with nodal negative squamous vulvar cancer
}

\author{
Florian Ebner ${ }^{1 *}$, Antje Beyer ${ }^{1}$, Amelie Schramm ${ }^{1}$, Andreas Rempen ${ }^{2}$, Thomas Blankenstein ${ }^{3}$, Julia Jückstock ${ }^{3}$, Thomas W.P. Fried1 ${ }^{1}$, Wolf- \\ gang Janni ${ }^{1}$, and Nikolaus de Gregorio ${ }^{1}$ \\ ${ }^{1}$ Department of Obstetrics and Gynecology, University Hospital Ulm, Prittwitzstr. 43, 89075 Ulm, Germany \\ ${ }^{2}$ Department of Obstetrics and Gynecology, Diakonie Schwäbisch Hall, Diakoniestr. 10, 74523 Schwäbisch Hall, Germany \\ ${ }^{3}$ Department of Obstetrics and Gynecology, Ludwig-Maximilians-University Munich, Maistr. 11, 80337 Munich, Germany
}

\begin{abstract}
Sentinel lymph node biopsy (SLNB) can reduce postoperative morbidity in women with vulvar cancer but data on long-term outcome of SLNB compared to inguinal lymph node dissection (ILND) is rare. Recurrence rates, disease-free survival (DFS) and overall survival (OS) from pT1/T2 pN0 squamous vulvar cancer from 1992 - 2011 were retrospectively compared between patients with SLNB ( $\mathrm{n}=56)$ or ILND ( $\mathrm{n}=56)$. PT2 tumors (59\% vs. 11\%; $\mathrm{p}<0.001)$ and complete vulvectomy (39\% vs. $4 \% ; \mathrm{p}<0.001$ ) occured more often in the ILND than the SLNB group with no difference in lymph node recurrence rate. Multivariate survival analysis considering the year of primary diagnosis, tumor stage and surgery revealed no significant effect of treatment on DFS ( $\mathrm{p}=0.062$ ) or OS ( $\mathrm{p}=0.924$ ). SLNB seems to be a safe treatment for women with pT1/T2 nodal-negative vulvar cancer with no difference in DFS or OS compared to ILND. Those results confirm the results of the GROINSS-V Study and other literature with the longest follow-up data reported within a real-life collective.
\end{abstract}

\section{Impact statement}

SLNB has been shown to reduce postoperative morbidity in women with vulvar cancer, however, data on long-term outcome of SLNB in vulvar cancer is rare. The aim of this study was to evaluate the outcome of patients with nodal-negative vulvar cancer that had undergone either SLNB or ILND in a clinical collective. Patients in the ILND group presented with more pT2 tumors (59\% vs. $11 \%$; p $<$ 0.001 ) and followed by complete vulvectomy (39\% vs. $4 \%$; $\mathrm{p}<0.001$ ). The lymph node recurrence rate was not significantly different between both groups $(\mathrm{p}=0.57)$. A multivariate survival analysis accounting for year of primary diagnosis, tumor stage and type of surgery revealed no significant effect of treatment on DFS or OS. SLNB seems to be a safe treatment for women with T1/T2 nodal-negative vulvar cancer, as we observed no difference in DFS or OS compared to patients with ILND. However, because of the observed bias between the two groups, results have to be interpreted carefully and need to be confirmed in larger prospective trials. Nevertheless, the results represent further data regarding the oncological safety of SNB supporting current literature and strongly underlie its value in the described setting with the longest follow-up, to date, reported.

\section{Introduction}

In vulvar cancer, the presence of lymph node metastases is the most important prognostic factor for local recurrence and survival $[1,2]$. Therefore, standard of care for vulvar cancer is surgical treatment with vulvectomy or wide radical excision of the primary tumor +/complete inguinal lymph node dissection (ILND). However, ILND is often accompanied by surgical complications such as impaired wound healing, acute infections, and lymph edema $[3,4]$. Even with advanced surgical techniques [5], more than half of the patients are affected by post-operative complications. In order to reduce the incidence of lymph edema, several attempts have been made to identify tumor infiltrated lymph nodes using non-invasive methods. Presurgical imaging with PET-CT [6], ultrasound [7] or MRI Bipat, et al. [8] and Kataoka, et al. [9] have been evaluated but did not reach high sensitivity and specificity required for accurate assessment. The concept of sentinel lymph node biopsy (SLNB) offers a way to reduce surgical morbidity, treatment costs $[10,11]$ and various studies in vulvar cancer have shown that it is safe and highly accurate in identifying lymph node metastases [1216]. Widely accepted eligibility criteria for performing a SNLB are: all tumor stages $>\mathrm{cT} 1 \mathrm{a}$ with a tumor diameter $<4 \mathrm{~cm}$, clinical negative groin lymph nodes, unifocal disease and surgical experience with the procedure. Further efforts to optimize identification of the SLN $[17,18]$ led to the conclusion that SLNB with technetium-based tests might reduce the need for ILND by up to $70 \%$ in women with early vulvar cancer [19].

However, there is still a lack of data regarding long-term outcome of patients with SLNB in terms of recurrence rates, disease-free survival and overall survival.

Correspondence to: Florian Ebner, Department of Obstetrics and Gynecology, University Hospital Ulm, Prittwitzstr. 43, 89075 Ulm, Germany, Tel: +49 751500 58554; Fax: +49 751500 58502; E-Mail: florian.ebner@uniklinik-ulm.de

Key words: vulvar cancer, inguinal lymph node dissection, sentinel lymph node biopsy, recurrence rate, survival

Received: March 02, 2017; Accepted: March 20, 2017; Published: March 22, 2017 
The aim of this study was to compare outcome in terms of recurrence rates and survival between patients with T1/T2 nodalnegative vulvar cancer who had undergone either SLNB (pN0sentinel -sn) alone or complete ILND (pN0).

\section{Methods}

Between 1992 and 2011 the gynecological cancer center databases of the University Hospital Ulm, the Ludwig-Maximilians-University Munich and the Diakonieklinikum Schwäbisch Hall were searched for women with node-negative $\mathrm{pT} 1 / \mathrm{pT} 2$ vulvar cancer. If no adequate follow-up data was available, appropriate tumor registries were searched for additional data. Exclusion criteria were metastasis or other non-squamous histology (e.g. melanoma). The study was approved by the local ethic committee.

ILND was performed via groin incision, identifying the inguinal ligament and femoral vein and artery. Lymphatic tissue along these anatomical structures was resected. For identification of SLN, technetium 99 was used and blue dye added according to surgeons' choice and SLN was removed with a small groin incision. Vulvar surgery for tumor resection with free margins included wide excision, partial or complete vulvectomy. Adjuvant treatment, given only in a minority of cases, included radiation or radiochemotherapy.

For all identified patients, year of and age at primary diagnosis, body mass index $\left(\mathrm{BMI} ; \mathrm{kg} / \mathrm{m}^{2}\right)$, characteristics of primary tumor (TNM classification, histological grading, histological type), ASA score, number of lymph nodes removed, type of surgery (complete vulvectomy, partial vulvectomy, wide excision) and treatment with radiation therapy (yes, no) were noted.

Categorical variables were described based on absolute and relative frequencies. Continuous variables were described by reporting medians and ranges. Comparisons between both groups (SLNB only vs. ILND) were conducted using the Mann-Whitney U test for continuous variables and the chi-square test for categorical variables (or Fisher's exact test in case of cells with expected frequencies less than 5 in $2 \times 2$ cross tabulations). Univariate survival analyses for disease-free survival (DFS) and overall survival (OS) were carried out based on Kaplan-Meier estimates and log rank tests. Multivariate survival analyses adjusting for the simultaneous effect of co-variates were performed using Cox proportional-hazards regression models. All time-to-event intervals were measured from date of primary surgery to date of the event or date of the last adequate follow-up. Statistical analyses were performed with $\mathrm{IBM}^{\oplus}$ SPSS $^{\circ}$ Statistics version 21 ; all statistical tests were two-sided and $p$ values of less than 0.05 were regarded as significant.

\section{Results}

Our database contained 189 patients with primary pT1/T2 vulvar carcinomas. After excluding cases with incomplete or inconclusive data (see defined inclusion criteria or other factors like lost to followup or cases of sentinel node none-detection), 112 patients (56 SLNB; 56 ILNE) with pT1/2 node-negative squamous vulvar cancer were available for analysis. A baseline comparison between SLNB group and ILND group is shown in Table 1. There were no significant differences between the two groups regarding age at primary diagnosis, BMI, ASA score, histological grading, proportion that underwent bilateral sentinel node biopsies or node dissections and proportion receiving radiation therapy. However, in our sample the ILND group comprised a higher proportion of patients that were diagnosed with vulvar cancer before the year 2005 compared to the SLNB group (55\% vs. 9\%; p < 0.001). In addition, patients in ILND group had a significantly higher proportion of T2 tumors ( $59 \%$ vs. $11 \%$; p < 0.001$)$ and received more often a complete vulvectomy ( $39 \%$ vs. $4 \%$; $<<0.001$ ). In the SLNB group, not more than 3 SLNs were removed per side (median 2 removed lymph nodes for left groin, 1 removed lymph node for right groin) and the total number of SLNs removed for both sides combined ranged from 1 to 6 (median 3). In contrast, up to 21 lymph nodes per side and 36 lymph nodes overall were removed in the ILND group (median left or right groin: 8 lymph nodes; median both sides combined: 15 lymph nodes). Regarding histological grade, patients within the SNB group had lesser G3 tumors (8.9\% vs $17.9 \%)$. However, this was not statistically significant ( $\mathrm{p}$ 0.051).

During the follow-up period (median 57 months in SLNB and 92 months in ILND group), 16 out of 56 (29\%) patients in the SLNB group had a recurrence; this included $10(18 \%)$ patients with local recurrence, 8 (14\%) patients with inguinal lymph node recurrence (6 of these patients had a bilateral SLNB) and $2(4 \%)$ patients with distant metastases. In the ILND group 26 out of $56(46 \%)$ patients had a relapse, including $21(38 \%)$ patients with local recurrence, $6(11 \%)$ patients with an inguinal recurrence ( 2 of these patients had a bilateral ILND) and one (2\%) patient with distant metastases. Overall, 4 out of $56(7 \%)$ women died in SLNB group and in 3 of these 4 cases $(75 \%)$ death was due to vulvar cancer. In the ILND group 17 out of $56(30 \%)$ women died, with 7 of these deaths $(41 \%)$ due to vulvar cancer. Rate of any recurrence was significantly higher in the ILND group $(p=0.051)$ and rates of local recurrence and death were significantly higher in the ILND group ( $p=0.020, p=0.002$, respectively; Figure 1 ), but there were no significant differences between both groups regarding rates of inguinal lymph node recurrence or distant metastases $(\mathrm{p}=0.568, \mathrm{p}=$ 1.000; Figure 1).

In $33(79 \%)$ of the 42 patients with a recurrence, surgery was performed; 17 (41\%) and 6 (14\%) patients with recurrences received radiotherapy or chemotherapy, respectively. There were no significant differences between the groups for proportion of patients with recurrences that received surgery $(\mathrm{p}=0.691)$, radiotherapy $(\mathrm{p}=0.389)$, or chemotherapy $(\mathrm{p}=1.000)$.

Univariate survival analysis showed no significant difference between the SLNB and the ILND group for both DFS (log rank test; $\mathrm{p}=$ 0.369 ; Figure 2 ) and OS (log rank test; $p=0.146$; Figure 3 ). Multivariate survival analysis accounting for date of primary diagnosis (before 2005 vs. 2005 or later), tumor stage (pT1, pT2) and type of surgery (complete vulvectomy, partial vulvectomy, wide excision) using cox regressions also revealed no significant difference between the treatment group (ILND vs. SLNB) on DFS (hazard ratio (HR) 2.07; 95\% confidence interval (CI) $0.97-4.46$; $\mathrm{p}=0.062$ ), though there was a tendency for shorter DFS in the ILND group. Likewise, multivariate cox regressions showed no significant effect of treatment group (ILND vs. SLNB) on OS (HR 0.93; 95\% CI $0.23-3.77 ; \mathrm{p}=0.924$ ). However, OS was related to tumor stage, as patients with pT2 tumors had a significantly shorter OS compared to patients with pT1 tumors (HR 3.90; 95\% CI 1.25 $12.11 ; \mathrm{p}=0.019)$. The interaction term between treatment group and tumor stage was not significant neither for DFS nor OS $(\mathrm{p}=0.267$ and $\mathrm{p}=0.223$, respectively).

Even if multivariate analyses accounted for the large discrepancies between the two treatment groups for tumor stage and interaction terms between treatment group and tumor stage were not significant for DFS and OS, we conducted additional subgroup analyses by performing multivariate cox regressions adjusted for date of primary 


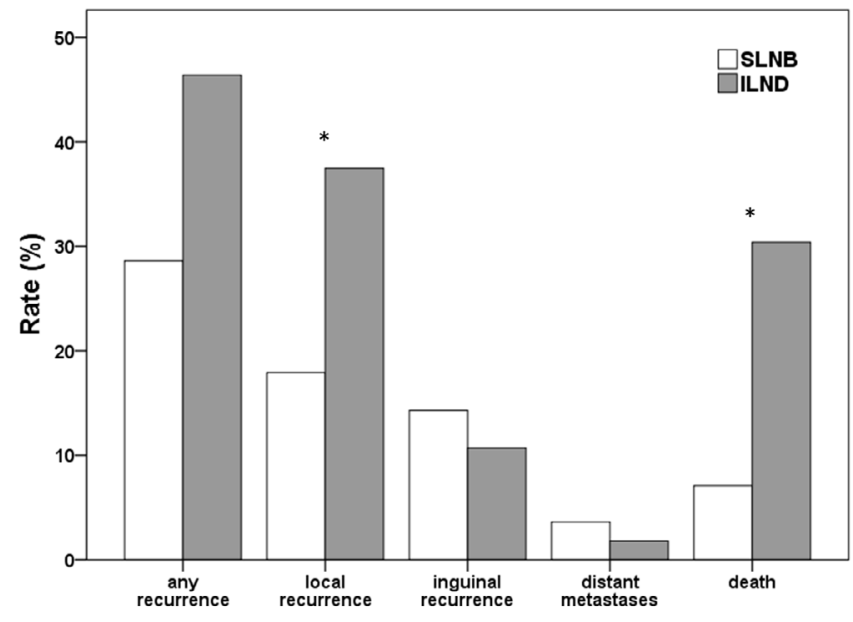

Figure 1. Rate of recurrences and deaths in patients with $\mathrm{pT} 1 / \mathrm{pT} 2$ nodal-negative squamous vulvar cancer that had undergone either SLNB alone (white bars) or ILND (grey bars). Significant differences between the two treatment groups are indicated with an asterisk.

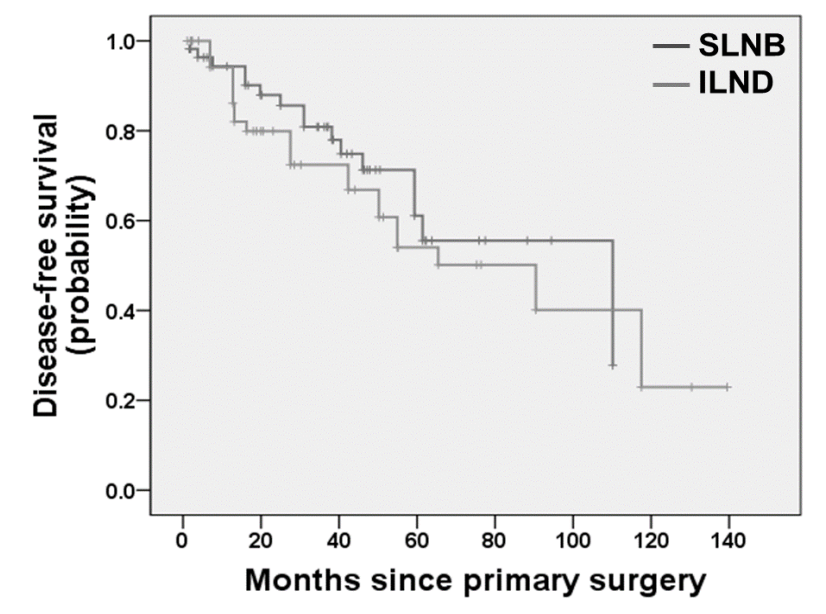

Figure 2.Kaplan-Meier plot for disease-free survival of patients with pT1/pT2 nodalnegative squamous vulvar cancer that had undergone either SLNB alone or ILND.

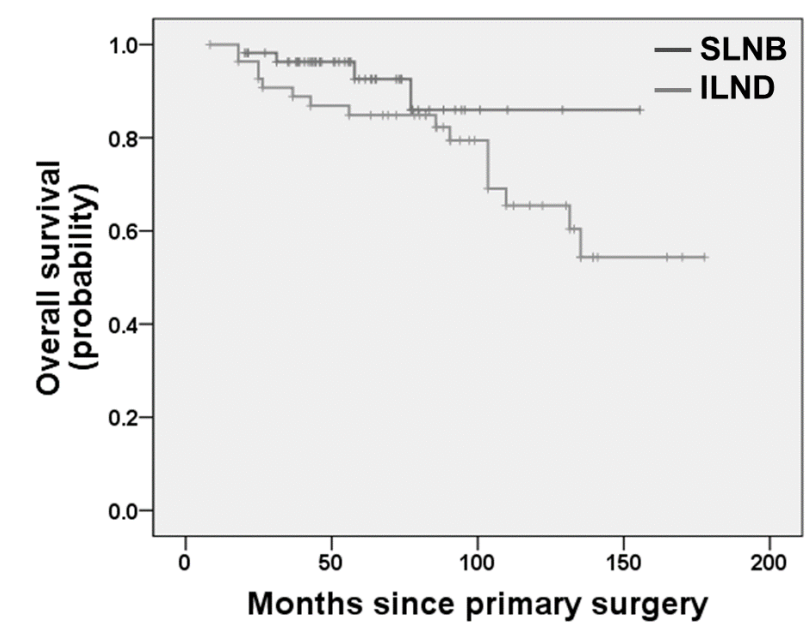

Figure 2.Kaplan-Meier plot for disease-free survival of patients with pT1/pT2 nodalnegative squamous vulvar cancer that had undergone either SLNB alone or ILND.
Table 1. Patient and tumor characteristics at baseline according to surgical treatment of lymph nodes (SLNB, sentinel lymph node biopsy; ILND, inguinal lymph node dissection)

\begin{tabular}{|c|c|c|c|c|}
\hline Variable & $\begin{array}{c}\text { Total } \\
\mathbf{N}=112\end{array}$ & $\begin{array}{l}\begin{array}{l}\text { SLNB group } \\
\quad \mathrm{N}=56\end{array}\end{array}$ & $\begin{array}{l}\text { ILND group } \\
\quad \mathrm{N}=56\end{array}$ & $p$-value \\
\hline $\begin{array}{c}\text { Age at primary } \\
\text { diagnosis(years) } \\
\text { Median } \\
\text { Range }\end{array}$ & $\begin{array}{c}69.0 \\
34-87\end{array}$ & $\begin{array}{c}68.5 \\
35-85\end{array}$ & $\begin{array}{c}69.0 \\
34-87\end{array}$ & $0.612^{1}$ \\
\hline $\begin{array}{c}\mathrm{BMI}\left(\mathrm{kg} / \mathrm{m}^{2}\right) \\
\text { Median } \\
\text { Range }\end{array}$ & $\begin{array}{c}25.8 \\
17.6-42.2\end{array}$ & $\begin{array}{c}25.2 \\
17.6-42.2\end{array}$ & $\begin{array}{c}26.1 \\
19.2-35.3\end{array}$ & $0.883^{1}$ \\
\hline $\begin{array}{c}\text { Year of primary diagnosis } \\
\text { Before } 2005 \\
2005 \text { or later }\end{array}$ & $\begin{array}{l}36(32.1 \%) \\
76(67.9 \%)\end{array}$ & $\begin{array}{c}5(8.9 \%) \\
51(91.1 \%)\end{array}$ & $\begin{array}{l}31(55.4 \%) \\
25(44.6 \%)\end{array}$ & $<0.001^{2}$ \\
\hline $\begin{array}{c}\text { ASA score } \\
1 \\
2 \\
3 \\
\text { Unknown }\end{array}$ & $\begin{array}{l}11(9.8 \%) \\
46(41.1 \%) \\
19(17.0 \%) \\
36(32.1 \%)\end{array}$ & $\begin{array}{l}9(16.1 \%) \\
24(42.9 \%) \\
12(21.4 \%) \\
11(19.6 \%)\end{array}$ & $\begin{array}{c}2(3.6 \%) \\
22(39.3 \%) \\
7(12.5 \%) \\
25(44.6 \%)\end{array}$ & $0.183^{2}$ \\
\hline $\begin{array}{c}\text { Tumor stage } \\
p T 1 \\
p T 2 \\
\text { Unknown }\end{array}$ & $\begin{array}{c}72(64.3 \%) \\
39(34.8 \%) \\
1(0.9 \%)\end{array}$ & $\begin{array}{c}49(87.5 \%) \\
6(10.7 \%) \\
1(1.8 \%)\end{array}$ & $\begin{array}{c}23(41.1 \%) \\
33(58.9 \%) \\
0(0.0 \%)\end{array}$ & $<0.001^{2}$ \\
\hline $\begin{array}{c}\text { Histological grading } \\
\text { G1 } \\
G 2 \\
G 3 \\
\text { Unknown }\end{array}$ & $\begin{array}{l}19(17.0 \%) \\
60(53.6 \%) \\
15(13.4 \%) \\
18(16.1 \%)\end{array}$ & $\begin{array}{c}14(25.0 \%) \\
29(51.8 \%) \\
5(8.9 \%) \\
8(14.3 \%)\end{array}$ & $\begin{array}{c}5(8.9 \%) \\
31(55.4 \%) \\
10(17.9 \%) \\
10(17.9 \%)\end{array}$ & $0.051^{2}$ \\
\hline $\begin{array}{l}\text { Type of surgery } \\
\text { Complete vulvectomy } \\
\text { Partial vulvectomy } \\
\text { Local excision }\end{array}$ & $\begin{array}{l}24(21.4 \%) \\
65(58.0 \%) \\
23(20.5 \%)\end{array}$ & $\begin{array}{c}2(3.6 \%) \\
41(73.2 \%) \\
13(23.2 \%)\end{array}$ & $\begin{array}{l}22(39.3 \%) \\
24(42.9 \%) \\
10(17.9 \%)\end{array}$ & $<0.001^{2}$ \\
\hline $\begin{array}{c}\text { Number of lymph nodes } \\
\text { removed }^{4} \\
\text { Median } \\
\text { Range }\end{array}$ & $\begin{array}{c}5.5 \\
1-36\end{array}$ & $\begin{array}{c}3 \\
1-6\end{array}$ & $\begin{array}{c}15 \\
4-36\end{array}$ & $<0.001^{1}$ \\
\hline $\begin{array}{c}\text { Bilateral SLNB or ILND } \\
\text { No } \\
\text { Yes } \\
\text { Unknown }\end{array}$ & $\begin{array}{c}28(25.0 \%) \\
74(66.1 \%) \\
10(8.9 \%)\end{array}$ & $\begin{array}{c}13(23.2 \%) \\
40(71.4 \%) \\
3(5.4 \%)\end{array}$ & $\begin{array}{c}15(26.8 \%) \\
34(60.7 \%) \\
7(12.5 \%)\end{array}$ & $0.492^{2}$ \\
\hline $\begin{array}{c}\text { Radiotherapy } \\
\text { No } \\
\text { Yes } \\
\text { Unknown }\end{array}$ & $\begin{array}{c}99(88.4 \%) \\
4(3.6 \%) \\
9(8.0 \%)\end{array}$ & $\begin{array}{l}49(87.5 \%) \\
2(3.6 \%) \\
5(8.9 \%)\end{array}$ & $\begin{array}{c}50(89.3 \%) \\
2(3.6 \%) \\
4(7.1 \%)\end{array}$ & $1.000^{3}$ \\
\hline
\end{tabular}

${ }^{1}$ Mann-Whitney U test

${ }^{2}$ Chi-square test, without unknowns

${ }^{3}$ Fisher's exact test

${ }^{4}$ sentinel lymph nodes only in the SLNB group

diagnosis and type of surgery separately for $\mathrm{pT} 1$ and $\mathrm{pT} 2$. Subgroup analyses showed that in pT1 tumors ILND was significantly associated with shorter DFS compared to SLNB (HR 2.63, 95\% CI $1.00-6.91 ; \mathrm{p}=$ 0.050 ), while there was no significant effect of treatment group (ILND vs. SLNB) on OS (HR 2.26, 95\% CI $0.29-17.79 ; \mathrm{p}=0.438$ ). In pT2 tumors, there was no significant effect of treatment group on DFS (HR $2.29,95 \%$ CI $0.41-12.91 ; \mathrm{p}=0.349$ ) and OS (HR 0.24, 95\% CI $0.03-$ $2.27 ; \mathrm{p}=0.213$.

\section{Discussion}

The SLNB concept offers a way to determine lymph node status without the surgical morbidities of a complete ILND. However, there is only data on long-term safety of the SLNB procedure from one randomized trial within a highly selected study collective [20]. Our retrospective study, which is one of the largest studies regarding patient numbers and follow up time, confirms oncological safety of SLNB, as it shows non-inferior survival for patients with node-negative vulvar cancer that have undergone SLNB compared to patients with complete ILND. Thus, results of this study might help spare patients the high morbidity associated with complete ILND. 
The SLNB concept has been adapted from other malignancies, reducing surgical mortality with slightly higher groin recurrence rates [15]. Lymphedema is one of the major complications after a ILND effecting up to $70 \%$ of the patients $[20,21]$. With removal of only SLN, lymph-edema rates dropped from 25.2 to 1.9 percent [20]. In a survey from $2010,73 \%$ of the surgeons stated that they have experience with the SLNB technique. However, despite the clear clinical benefit, surgeons have not yet widely adopted this concept as standard of care, in early vulvar cancer [22]. Contrary to a study comparing quality of life between vulvar cancer patients after SLNB only and ILND showed that the majority of patients with SLNB are more content with their overall status and would advise the SLNB-procedure to relatives [23]. Another study reported that most women would choose complete ILND over SLNB, preferring the - 'assumed' - higher safety of ILND over improved quality of life [24]. These studies suggest that the reasons behind the low acceptance of SLNB amongst patients and surgeons might be the lack of data regarding long-term oncological safety.

However, there are several retrospective studies for oncological safety indicating that there is no difference in oncological outcome between SLNB and ILND [14,20,25,26]. Johann, et al. [14] compared the outcome between 16 patients with pN0 pT1/2 after SLNB and 18 patients with ILND [14]. After a follow up of 24 months no groin recurrences were found. The largest multicenter trial was published by Van der Zee, et al. [20]. Here, 259 patients with negative SLNB, $97 \%$ unifocal disease and a median follow up of 35 months had a recurrence rate of $2.3 \%$ and an 3 year survival rate of $97 \%$. The authors conclude that SLNB is an oncological safe treatment option for early, unifocal vulvar cancers that should be discussed with the patient. A prospective observational study with a median follow up of 58 months in 57 N0 (sn) patients reported a groin recurrence rate of 5.2\% [27]. Thus far, this has been the longest reported follow up in a prospective study on SLNB and vulvar cancer. Even though no control group was followed in the study, the recurrence rate is considered comparable to the rate in patients with ILND.

Our results underline the oncological safety of the SLNB in early vulvar cancer, however conclusive evidence could only be provided by a large randomized clinical trial with long follow up. Organising a prospective randomized trial with adequate patient numbers is considered very demanding by various national organisations [20]. As vulvar cancer is a rare condition and early stages have a good prognosis [28], numbers needed for such a study are unlikely to be recruited. Nevertheless, a survey conducted by the Study Group for Gynecological Oncology (AGO) indicated the willingness of physicians to participate in such a prospective trial [22].

Prospective but not randomized trials already have shown a low inguinal recurrence rate for SLNB [27], underlining the feasibility of the method [12]. Another publication prospectively looked at the quality of life after inguinal surgery [29] and authors found a clear benefit for a less radical approach.

There are some major weaknesses of our study that need to be addressed. While the two treatment groups did not differ significantly with regard to age at primary diagnosis, BMI, grade, proportion with bilateral SLNB or ILND and radiotherapy, patients of the ILND group had larger tumors and more often a complete vulvectomy. The reason for these observed differences is probably that SLNB techniques are more likely offered and agreed on by patients with small tumors, which can also more often be removed with wide excision rather than vulvectomy. Another difference between both groups was that in the ILND group a higher proportion of patients was diagnosed with vulvar cancer before the year 2005, which is due to the fact that SLNB became a recognized option in vulvar cancer only in recent years. As a consequence, median follow-up was considerably longer in the ILND group, which is reflected by a higher observed recurrence and death rates. Even if these differences were accounted for in our multivariate survival analysis by adjusting for tumor size, type of surgery and year of primary diagnosis, results (i.e., no significant effect of treatment group on DFS or OS) have to be interpreted carefully. Another limitation is that data on focality, localization/laterality of primary tumor and/or recurrences, resection margins and lymphovascular space invasion were not consistently recorded in a way that these variables could be included as factors in our analysis. Subgroup analyses with pT1 tumors in ILND were significantly (albeit barely) associated with shorter DFS (HR 2.63, 95\% CI 1.00 - 6.91; $\mathrm{p}=0.050$ ) underlying the suggestion that other, here not accounted for, prognostic factors did also contribute.

While our local recurrence rate was high but comparable to existing data (e.g. the 10 year follow up of the GROINSS-V Study) [30], our inguinal recurrence rate was higher than reported. This might potentially be reflecting a real life collective, where preoperative diagnostics were done according to physicians' choice and a histological workup was routine, in contrast to the Dutch trial with a sophisticated preoperative workup including ultrasound and a second pathologist analyzing all histologically negative SLNs. The higher death rate in the ILND group is explained by the longer follow of due to the already advanced age of patients at diagnosis, only $41 \%$ of patients did die from vulvar cancer.

In summary, our data support the use of SLNB in early vulvar cancer as we found no significant differences in DFS or OS between patients with nodal-negative vulvar cancer that had undergone either SLNB only or ILND. However, results need to be confirmed in a large prospective study. Our data supports the GROINSS-V Study even with the longest reported follow-up.

\section{Disclosure statement}

All authors report no declarations of interest.

\section{References}

1. Linn W, Fabian T, Lilli K, Donata G, Cordula P, et al. (2013) Management of patients with vulvar cancer: a perspective review according to tumour stage. Ther Adv Med Oncol 5: 183-192.

2. Baiocchi V, Dominico D, Ferlito R, Giannonc F, Guarascio M, et al. (2013) Test of a building velnerability model for L'Aquila earthquake. Applied Genomics 4: 95-103.

3. Ercole CE, Pow-Sang JM, Spiess PE (2013) Update in the surgical principles and therapeutic outcomes of inguinal lymph node dissection for penile cancer. Urol Oncol 31: 505-516.

4. Berger J, Scott E, Sukumvanich P, Smith A, Olawaiye A, et al. (2015) The effect of groin treatment modality and sequence on clinically significant chronic lymphedema in patients with vulvar carcinoma. International journal of gynecological cancer?: $O \mathrm{~J}$ Intern Gyn Can Soc 25: 119-24.

5. Abbas S, Seitz M (2011) Systematic review and meta-analysis of the used surgica techniques to reduce leg lymphedema following radical inguinal nodes dissection. Surgical oncology 20: 88-96.

6. Kamran MW, O'Toole F, Meghen K, Gleeson N (2014) Whole-body [18F]fluoro-2deoxyglucose positron emission tomography scan as combined PET-CT staging prior to planned radical vulvectomy and inguinofemoral lymphadenectomy for squamous vulvar cancer: a correlation with groin node metastasis. Eur J Gynaecol Oncol 35: 230-235.

7. de Gregorio N, Ebner F, Schwentner L, Friedl TW, Deniz M, et al. (2013) The role of preoperative ultrasound evaluation of inguinal lymph nodes in patients with vulvar malignancy. Gynecol Oncol 131: 113-117. [Crossref] 
8. Bipat S, Fransen GA, Spijkerboer AM, van der Velden J, Bossuyt PM, et al. (2006) Is there a role for magnetic resonance imaging in the evaluation of inguinal lymph node metastases in patients with vulva carcinoma? Gynecol Oncol 103: 1001-1006.

9. Kataoka MY, Sala E, Baldwin P, Reinhold C, Farhadi A, et al. (2010) The accuracy of magnetic resonance imaging in staging of vulvar cancer: a retrospective multi-centre study. Gynecol Oncol 117: 82-87. [Crossref]

10. McCann GA, Cohn DE, Jewell EL, Havrilesky LJ (2014) Lymphatic mapping and sentinel lymph node dissection compared to complete lymphadenectomy in the management of early-stage vulvar cancer: A cost-utility analysis. Gynecol Oncology 136: 300-304. [Crossref]

11. Erickson BK, Divine LM, Leath CA, Straughn JM Jr (2014) Cost-effectivenes analysis of sentinel lymph node biopsy in the treatment of early-stage vulvar cancer. $J$ Inter Gynecol Cancer Soc 24: 1480-1485.

12. de Hullu JA, Hollema H, Piers DA, Verheijen RH, van Diest PJ, et al. (2000) Sentinel lymph node procedure is highly accurate in squamous cell carcinoma of the vulva. $J$ Clin Oncol 18: 2811-2816. [Crossref]

13. Oonk MH, Hollema H, de Hullu JA, van der Zee AG (2006) Prediction of lymph node metastases in vulvar cancer: a review. O J Intern Gynecol Canc Soc 16: 963-971.

14. Johann S, Klaeser B, Krause T, Mueller MD (2008) Comparison of outcome and recurrence-free survival after sentinel lymph node biopsy and lymphadenectomy in vulvar cancer. Gynecol Oncol 110: 324-328.

15. Meads C, Sutton AJ, Rosenthal AN, Maaysiak S, Kowalska M, et al. (2014) Sentinel lymph node biopsy in vulval cancer: systematic review and meta-analysis. Br J Cancer 110: 2837-2846. [Crossref]

16. Vidal-Sicart S, Puig-Tintoré LM, Lejárcegui JA, Pons F, Ortega ML, et al. (2007) Validation and application of the sentinel lymph node concept in malignant vulvar tumours. Eur J Nucl Med Molecul Imag 34: 384-391.

17. Hampl M, Hantschmann P, Michels W, Hillemanns P (2008) Validation of the accuracy of the sentinel lymph node procedure in patients with vulvar cancer: results of a multicenter study in Germany. Gynecol Oncol 111: 282-288. [Crossref]

18. Handgraaf HJ, Verbeek FP, Tummers QR, Boogerd LS, van de Velde CJ, et al. (2014) Real-time near-infrared fluorescence guided surgery in gynecologic oncology: a review of the current state of the art. Gynecol Oncol 135: 606-613. [Crossref]

19. Lawrie TA, Patel A, Martin-Hirsch PP, Bryant A, Ratnavelu ND, et al. (2014) Sentinel node assessment for diagnosis of groin lymph node involvement in vulval cancer. Cochrane Database Syst Rev : CD010409. [Crossref]
20. Van der Zee AG, Oonk MH, De Hullu JA, Ansink AC, Vergote I, et al. (2008) Sentinel node dissection is safe in the treatment of early-stage vulvar cancer. J Clin Oncol 26 : 884-889. [Crossref]

21. Gaarenstroom KN, Kenter GG, Trimbos JB, Agous I, Amant F, et al. (2003) Postoperative complications after vulvectomy and inguinofemoral lymphadenectomy using separate groin incisions. J Inter Gynecol Cancer Soc 13: 522-527. [Crossref]

22. Kramer F, Hertel H, Hillemanns P (2013) Use of the Sentinel Lymph Node Technique Compared to Complete Inguino-femoral Lymph Node Removal in Patients with Invasive Vulvar Cancer in Germany. Geburtshilfe Frauenheilkd 73: 142- 147.

23. Oonk MH, van Os MA, de Bock GH, de Hullu JA, Ansink AC, et al. (2009) A comparison of quality of life between vulvar cancer patients after sentinel lymph node procedure only and inguinofemoral lymphadenectomy. Gynecol Oncol 113: 301-305. [Crossref]

24. Farrell R, Gebski V, Hacker NF (2014) Quality of life after complete lymphadenectomy for vulvar cancer: do women prefer sentinel lymph node biopsy? J Inter Gynecol Cancer Soc 24: 813-819.

25. Canlorbe G, Rouzier R, Bendifallah S, Chéreau E (2012) Impact of sentinel node technique on the survival in patients with vulvar cancer: analysis of the Surveillance, Epidemiology, and End Results (SEER) database. Gynécologie, obstétrique \& fertilité 40: 647-651.

26. Terada KY, Shimizu DM, Jiang CS, Wong JH (2006) Outcomes for patients with T1 squamous cell cancer of the vulva undergoing sentinel node biopsy. Gynecol Oncol 102: 200-203. [Crossref]

27. Robison K, Roque D, McCourt C, Stuckey A, DiSilvestro PA, et al. (2014) Long-term follow-up of vulvar cancer patients evaluated with sentinel lymph node biopsy alone. Gynecol Oncol 133: 416-420. [Crossref]

28. Maggino T, Landoni F, Sartori E, Zola P, Gadducci A, et al. (2000) Patterns of recurrence in patients with squamous cell carcinoma of the vulva. A multicenter CTF Study. Cancer 89: 116-122. [Crossref]

29. Novackova M (2015) A prospective study in the evaluation of quality of life after vulvar cancer surgery. International journal of gynecological cancer? OJ Intern Gynecol Canc Soc 25: 166-173.

30. te Grootenhuis NC, van der Zee AG, van Doorn HC, van der Velden J, Vergote I, et al. (2016) Sentinel nodes in vulvar cancer: Long-term follow-up of the GROningen INternational Study on Sentinel nodes in Vulvar cancer (GROINSS-V) I. Gynecologic Oncology 140: 8-14. [Crossref]

Copyright: (C2017 Ebner F. This is an open-access article distributed under the terms of the Creative Commons Attribution License, which permits unrestricted use, distribution, and reproduction in any medium, provided the original author and source are credited. 